\title{
A Simulation Study on Beam Bunching in the KEK 2.5-GeV Linac New Pre-Injector
}

\author{
T. Kamitani, J. - Y. Choi, A. Enomoto, S. Ohsawa, Y. Ogawa, T. Urano, T. Suwada, K. Furukawa and I. Sato \\ National Laboratory for High Energy Physics (KEK) \\ 1-1 Oho, Tsukuba-shi, Ibaraki-ken, 305, Japan
}

\begin{abstract}
We have performed a simulation study on beam bunching in the KEK 2.5-GeV linac new pre-injector comprising double prebunchers and a buncher. Dependence of the bunching performance upon the if powers and the phases of the prebunchers is discussed. We obtained an optimum bunch length of 4 ps with the simulation.
\end{abstract}

\section{INTRODUCTION}

The pre-injector of the KEK $2.5-\mathrm{GeV}$ linac was upgraded in the summer of 1992 [1]. The bunching system was improved in order to accelerate more intense electron beams required for the production of positrons for the KEK B-factory [2], which is under consideration as a future project of KEK. A short bunch is essential for the injection efficiency to the B-factory ring, since the bunch length of the electrons determines that of the positrons and it set the limit of the narrowness of the energy spectrum. Besides, the emittance degradation by the transverse wake-field is larger for a longer bunch. To achieve good bunching performance, we adopted a system comprising double prebunchers and a buncher. The system was designed to be flexible; the rf input power and the relative if accelerating phase of each buncher are independently tunable for optimum bunching. The parameters of the system are adjusted according to the change of the beam conditions such as the currents and the pulse length. The dependence of the bunch length upon the relative phases and the $\mathrm{rf}$ input powers of the prebunchers is studied using a beam dynamics simulation code.

\section{BUNCHING SYSTEM}

In bunching an intense beam, it is important to minimize the effect of the debunching due to space-charge forces. We thus adopted a system of double prebunchers and a buncher. The first prebuncher (PB1) provide the d-c beam from the gun with the velocity modulation and the beam is slightly bunched in the drift space to the second prebuncher (PB2). The if phase for PB2 is adjusted for the electrons to enter PB2 in accelerating phase, causing further bunching and acceleration as well. The debunching of the beam is significant at low energy, it is desirable to accelerate the electrons in earlier stage of the bunching. Acceleration of the electrons by PB2 before entering the buncher is effective for improving the bunching performance. The electrons are finally bunched in the strong accelerating field of the buncher.

A schematic diagram of the system is shown in Figure 1. Parameters of the bunchers are summarized in Table 1. All three bunchers are traveling-wave structures operated in the $2 \pi / 3$ mode of $2856 \mathrm{MHz}$. An array of the Helmholtz coils encompasses the bunchers. They produce a uniform solenoidal focusing field of $1.0 \mathrm{kG}$. A new high-power attenuator-and-phase-shifter system is used for the independent adjustment of the rf input power and the accelerating if phase [3]. This flexibility of the bunching system is expected to enhance the capability of the parameter tuning.

Table 1

Bunching system design values

Prebuncher l (PB1) maximum field available input power cavity number cavity size $D$

Prebuncher 2 (PB2) maximum field available input power cavity number

Buncher cavity size $D$

maximum field available input power cavity number buncher section normal section cavity size D

Focusing magnetic field Helmholtz coils

$$
\begin{aligned}
& 0.4 \mathrm{MV} / \mathrm{m} \\
& 0.1 \mathrm{MW} \\
& 7 \\
& 24.318 \mathrm{~mm} \\
& \\
& 2.0 \mathrm{MV} / \mathrm{m} \\
& 2.3 \mathrm{MW} \\
& 5 \\
& 24.318 \mathrm{~mm}
\end{aligned}
$$

$15 \mathrm{MV} / \mathrm{m}$ $13 \mathrm{MW}$

6

29

$27.01 \sim 34.99 \mathrm{~mm}$

$1.0 \mathrm{kG}$
We have estimated the performance of the system to search for the optimum values for these operation parameters with the simulation code PARMELA.

\section{SIMULATION}

The PARMELA code is widely used for designing bunching systems of electron linear accelerators [4]. In this code, an electron beam is represented by a few hundreds
PB1

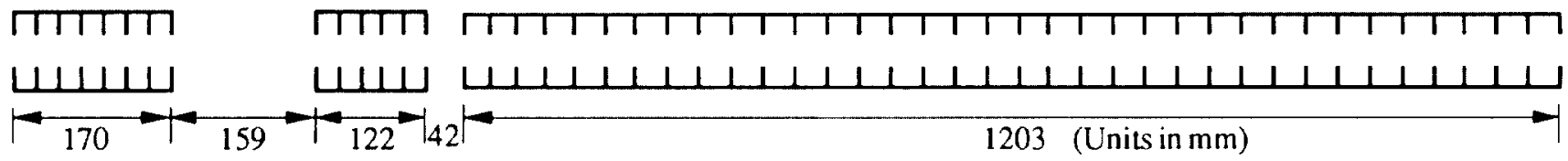

Figure. 1 Layout of the first and second prebuncher and the buncher 
( -300 in our calculations) of macroparticles. They are traced in consecutive finite time-steps under the influence of the accelerating electric fields, the focusing magnetic fields and the space-charge forces. Effects due to the wake-field in the cavities are not taken into account. As an initial condition, these macroparticles were assumed to be distributed randomly within the specified volume in the 4-dimensional transverse phase space. The initial transverse r.m.s. emittance was taken to be $7 \pi \mathrm{mm}$.mrad. In the longitudinal direction, they have a uniform distribution within the range corresponding to one wave length $\beta \lambda(=73.5 \mathrm{~mm})$ of the $2856 \mathrm{MHz}$. ff acceleration, where $\beta=v / c$. Their initial energies were 200 $\mathrm{keV}$. All the calculations were made for the beam current of $4 \mathrm{~A}$.

To estimate the bunching performance of our double prebuncher system, we have studied the dependence of the bunch lengths upon the if parameters of PB1 and PB2. The shapes of the bunches are dependent upon these parameters. In certain conditions, bunches have very irregular forms as shown in Figure 4(a). To discuss the bunch lengths of such irregular bunches, FWHM's or r.m.s. widths are not suitable. Instead, we used a fraction of the particles which lie in the rf phase width of 4 degrees around the peak of the distribution (Bunch Core). We chose this criterion because the optimum bunch width obtained by the calculations was about 4 degrees as shown later. This reflects well not only the sharpness of the bunch shapes but the extent of the tail or sub-peaks, regardless of forms of the bunches. We, therefore, used this criterion as an index evaluating the bunching performance in the following discussion.

In studying the dependence of the bunching performance upon the rf parameters, we took high and low field cases of PB1. The dependence upon the rf field and input phase of PB2 was mainly studied with those of the buncher fixed. Table 2 gives the range of the if fields varied in the calculations.

Table 2

Range of the rf fields

\begin{tabular}{lc} 
& Range of the rf fields \\
\hline PB1 & $0.15,0.40 \mathrm{MV} / \mathrm{m}$ \\
PB2 & $0.1 \sim 2.5 \mathrm{MV} / \mathrm{m}$ \\
Buncher & $15 \mathrm{MV} / \mathrm{m}$ \\
\hline
\end{tabular}

\section{RESULTS \& DISCUSSION}

The bunching performance is dependent not only on the electric field of the prebunchers but also their relative phases. The effect of their electric fields was studied first. The relative rf phase of PB2 to that of PB1 was fixed, such as the incident phase at PB2 to be 30 degrees for the reference particle which was at the zero phase in PB1. The incident phase at the buncher was also fixed to 50 degrees in the case that the field of PB2 is $2.0 \mathrm{MV} / \mathrm{m}$. Figure 2 shows the dependence of the bunching performance upon the electric field of PB2. It was evaluated with the index defined in the previous section.

It can be shown that the better performance is obtained for the weaker field $(0.15 \mathrm{MV} / \mathrm{m})$ of PB1 in the whole range in Figure 2. The optimum value of the electric field of PB2 is $2.0 \mathrm{MV} / \mathrm{m}$ for both cases.

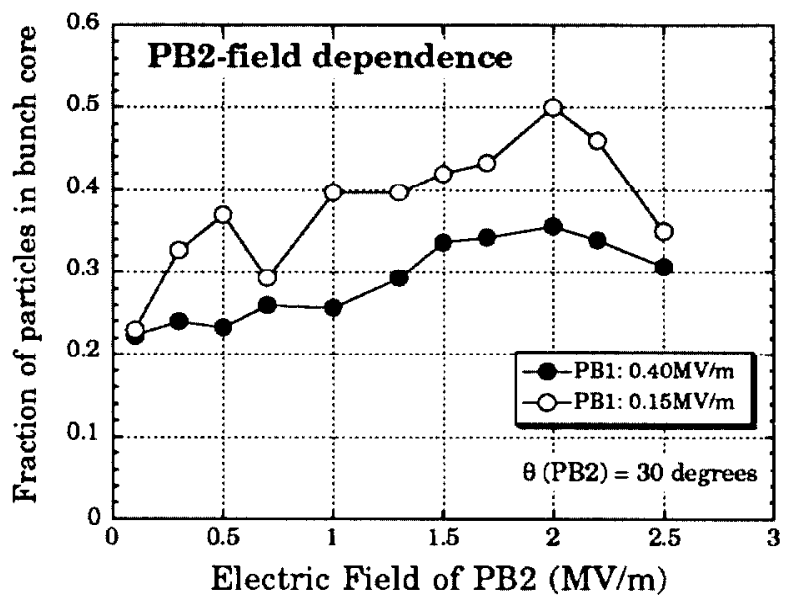

Figure 2. Dependence of the bunching performance upon the electric field of PB2.

Concerning the bunching performance upon the phase of PB2, we have studied for the cases of PB2's fields of 1.0 and $2.0 \mathrm{MV} / \mathrm{m}$ (Figure 3). The relative phase of the buncher to that of PB1 was not changed.

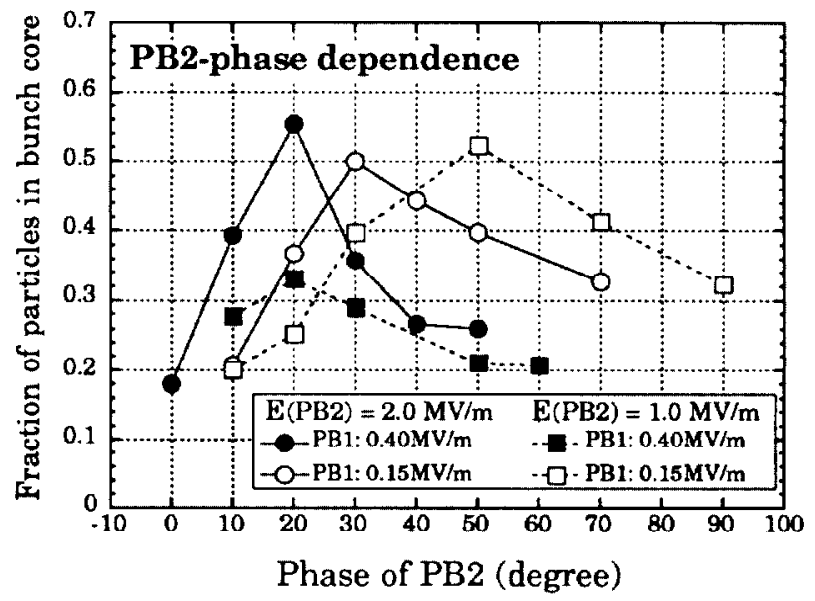

Figure 3. Dependence of the bunching performance upon the If phase of $\mathrm{PB} 2$.

For the weaker field $(0.15 \mathrm{MV} / \mathrm{m})$ of $\mathrm{PB} 1$, the good performances are obtaincd for the wide range of the phase of $\mathrm{PB} 2$. The range is narrower for the stronger field $(0.40$ $\mathrm{MV} / \mathrm{m}$ ) of PB1 and the two-peak structures of the bunches were observed for the conditions a little away from the optimum value as shown in Figure 4 (a). The similar structures were also observed in experiments as shown in Figure 4 (b). The structure is believed to be caused by the over-bunching due to the excessive field of PB1.

With the optimum condition obtained for the stronger field of PB1, the two peaks are expected to be coalesced. Calculated trajectories for this case are shown in Figure 5. It shows that the two sub-bunches, formed by the overbunching, coalesce in such a particular phase relation that the preceding sub-bunch is decelerated while the subsequent subbunch is accelerated in the rf field of PB2. The discontinuity of the trajectories shown in the figure is because the positions 
of the particles are expressed with the references to the if phase of each buncher. Though the condition gives a good performance, the tolerance of the relative phase of $\mathrm{PB} 2$ is small as shown in Figure 3.
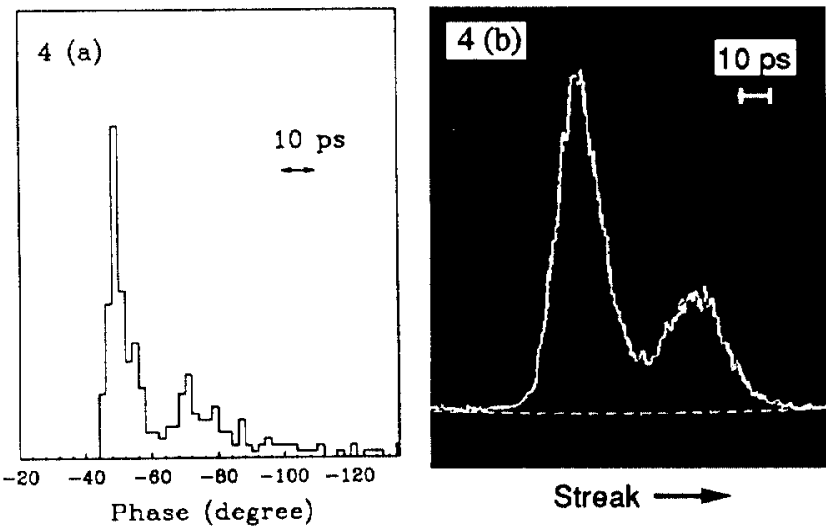

Figure 4. Bunches with two-peak structure by the calculation (a) and by the measurement (b) [5].

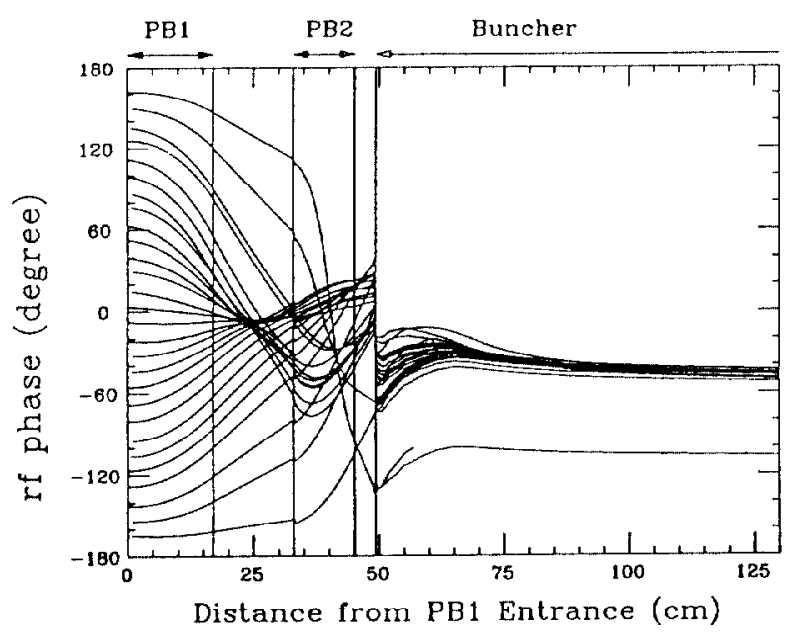

Figure 5. Typical trajectories for the optimum condition with the field of $0.4 \mathrm{MV} / \mathrm{m}$ for PB1.

For the optimum condition for the weaker field $(0.15$ $\mathrm{MV} / \mathrm{m}$ ) of PB1, calculated trajectories are shown in Figure 6. This optimum bunching results from the moderate bunching by PB1 and the further bunching by PB2 and the buncher. The bunch shape with this condition is shown in Figure 7. The FWHM bunch width is estimated to be about 4 degrees, which corresponding to the time duration of $4 \mathrm{ps}$.

\section{SUMMARY}

We have studied the bunching performances of our new bunching system with the simulation code PARMELA. The bunch length of $4 \mathrm{ps}$ is obtained as a preliminary optimum value. Further extensive optimization of the parameters with the simulation will be performed. The effects due to the longitudinal wake-field will be included there. The experimental studies measuring the bunch length with the optical transition radiation monitor [5] will also be performed.

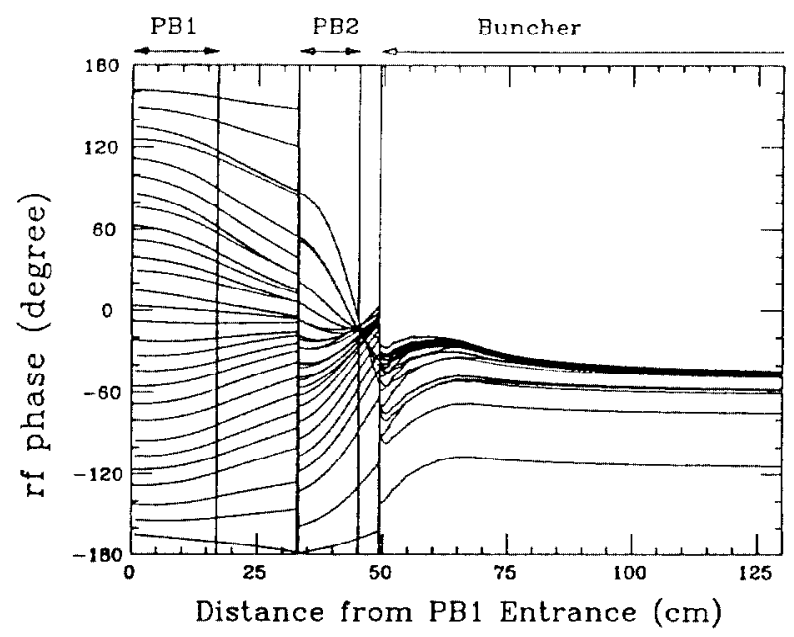

Figure 6. Typical trajectories for the optimum condition with the field of $0.15 \mathrm{MV} / \mathrm{m}$ for $\mathrm{PB} 1$.

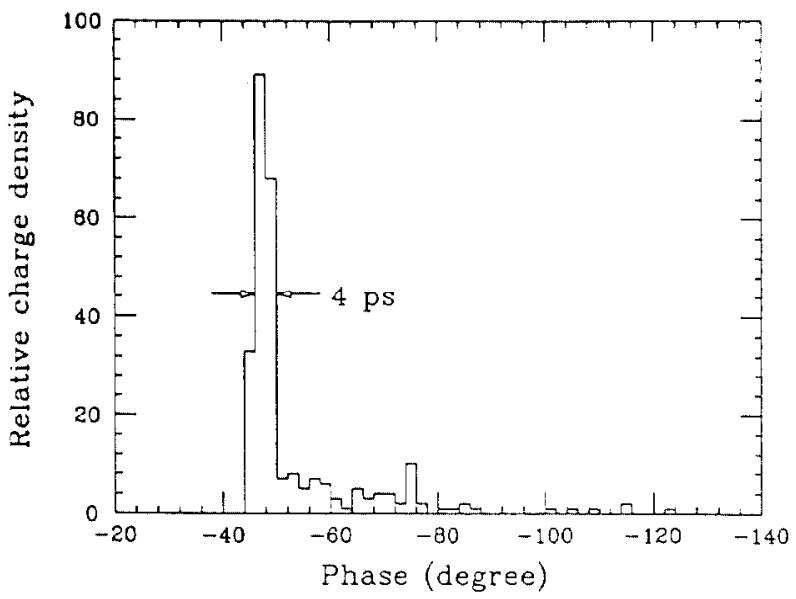

Figure 7. Bunch shape calculated for the optimum condition with the field of $0.15 \mathrm{MV} / \mathrm{m}$ for $\mathrm{PB}$.

\section{REFERENCES}

[1] S. Ohsawa et al., "Improvement to the Injection system of the KEK 2.5-GeV Linac," Conference Record of the 16 th International Linac Conference, 1992, pp. 91-93. S. Ohsawa et al., "New Pre-injector of the KEK 2.5GeV Linac and its Performance," these proceedings.

[2] A. Enomoto et al., "Linac Upgrade Plan for the KEK BFactory," these proceedings.

[3] S. Ohsawa et al., "High-Power Hybrid Attenuator \& Phase-Shifter Systems," Conference Record of the 15 th International Linac Conference, 1990, pp. 159-161.

[4] R. Miller et al., "Design of a Bunching System for a High Intensity Electron Linac," LBL-25237-mc, Jun. 1988. 3pp.; Proceedings of the first European Particle Accelerator Conf., Rome, Italy, Jun. 7-11, 1988.

[5] Y. Ogawa et al., "Beam Monitor Utilizing Transition Radiation," these proceedings. 